\title{
Fatores associados ao desenvolvimento mental e motor de crianças de quatro creches públicas de Recife, Brasil
}

\author{
Factors associated with mental and psychomotor development of infants in four public day care centers \\ in the municipality of Recife, Brazil
}

Sophie Helena Eickmann ${ }^{1}$, Adriana Maria S. Maciel², Pedro Israel C. Lira ${ }^{3}$, Marilia de Carvalho Lima ${ }^{4}$

\section{RESUMO}

Objetivo: Identificar os fatores associados aos índices de desenvolvimento mental e motor em lactentes que frequentam creches da rede municipal em Recife.

Métodos: Estudo de corte transversal realizado entre fevereiro e abril de 2005 em uma amostra de 108 crianças com idade entre quatro e 24 meses, pertencentes a quatro creches municipais do Recife. O desenvolvimento mental e motor foi avaliado pela Escala de Desenvolvimento Infantil de Bayley II. Por meio de entrevistas às mães, foram obtidas informações sobre as condições socioeconômicas e demográficas das famílias e as características biológicas das crianças ao nascer (peso, sexo e idade gestacional). O estado nutricional foi avaliado através dos índices peso/idade, comprimento/idade, peso/comprimento e da concentração de hemoglobina.

Resultados: As médias dos índices de desenvolvimento mental e motor foram $88 \pm 11$ e $95 \pm 12$ pontos, respectivamente. A idade gestacional apresentou associação significativa com o desenvolvimento mental e motor e o peso ao nascer e o estado nutricional atual, avaliado pelo índice peso/comprimento, se associaram ao desenvolvimento motor.

Conclusões: As crianças analisadas apresentaram desenvolvimento neuropsicomotor ainda na faixa de normalidade, mas aquém do esperado para a idade, comparado a populações de referência em países desenvolvidos. Os fatores associados ao baixo desempenho do desenvolvimento nessa população estão relacionados aos fatores biológicos, destacando-se os déficits nutricionais, cuja redução deve ser uma prioridade para gestores em saúde e educação.
Palavras-chave: desenvolvimento infantil; creches; recém-nascido de baixo peso; prematuro; estado nutricional; anemia.

\section{ABSTRACT}

Objective: To identify factors associated with mental and psychomotor development of infants enrolled at public day care centers of Recife, Pernambuco, Brazil.

Methods: This is a cross-sectional study conducted with 108 infants aged four to 24 months attending four public day care centers of the Municipality of Recife from February to April 2005. Mental and psychomotor development was assessed by the Bayley Scale of Infant Development II. Family socioeconomic and demographic conditions and infant biological characteristics at birth (weight, sex and gestational age) were obtained by maternal interview. Nutritional status was assessed by the indexes "weightfor-age", "length-for-age" and "weight-for-length" and by hemoglobin level.

Results: Mean mental and psychomotor development indexes were $88 \pm 11$ and $95 \pm 12$, respectively. Gestational age showed significant association with mental and psychomotor development. Birthweight and current nutritional status assessed by "weight-for-length" were associated with psychomotor development.

Conclusions: Mental and psychomotor development were in the the normal range, but, below the expected for infants of the same age group of reference populations in developed countries. The factors associated with poor developmental
Instituição: Universidade Federal de Pernambuco (UFPE), Recife, PE, Brasil ${ }^{1}$ Doutora em Nutrição; Professora adjunta do Departamento Materno Infantil da UFPE, Recife, PE, Brasil

${ }^{2}$ Mestre em Saúde da Criança e do Adolescente pela UFPE; Professora da Faculdade Integrada do Recife (FIR), Recife, PE, Brasil

${ }^{3}$ Doutor em Medicina; Professor associado do Departamento de Nutrição da UFPE, Recife, PE, Brasil

${ }^{4}$ Doutora em Medicina; Professora adjunta do Departamento Materno Infantil da UFPE, Recife, PE, Brasil

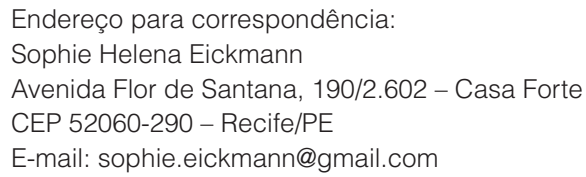

Endereço para correspondência:

Sophie Helena Eickmann

Avenida Flor de Santana, 190/2.602 - Casa Forte

CEP 52060-290 - Recife/PE

E-mail: sophie.eickmann@gmail.com

Fonte financiadora: Conselho Nacional de Desenvolvimento Científico e Tecnológico (CNPq), processo no 505572/2004-4.

Recebido em: 26/8/08

Aprovado em: 2/2/09 
indexes in this population are related to biological factors, with emphasis on nutritional deficits. Their reduction should be a priority for health and educational policy managers.

Key-words: child development; child day care centers; infant, low birth weight; infant, premature; nutritional status; anemia.

\section{Introdução}

Os dois primeiros anos de vida são fundamentais para o desenvolvimento infantil, ocorrendo rápido crescimento cerebral e intenso avanço cognitivo e sensório-motor, que podem ser influenciados por uma série de fatores biológicos e ambientais. Crianças de baixo nível socioeconômico são frequentemente expostas a múltiplos fatores adversos, constituindo um importante grupo de risco para atrasos no desenvolvimento ${ }^{(1-7)}$.

Em diversas regiões urbanas pobres do Brasil, crianças em situações sociais de risco costumam frequentar creches públicas, o que parece ser um fator de proteção para o seu estado nutricional, não sendo tal efeito, entretanto, um consenso entre os autores ${ }^{(8-10)}$. Por outro lado, também existem controvérsias sobre a influência da creche no desenvolvimento infantil, sobretudo no que diz respeito ao desenvolvimento cognitivo e comportamental. Alguns autores relatam que a creche funciona como um fator de proteção, principalmente para crianças que vivem em condições ambientais adversas; outros, porém, relatam um impacto negativo no desenvolvimento cognitivo e da linguagem em pré-escolares, sugerindo, ainda, que crianças que frequentam creches costumam ser mais agressivas com seus pares ${ }^{(11-18)}$.

A escassez de estudos e as controvérsias existentes sobre o tema, sobretudo nos países em desenvolvimento, motivaram a realização desta pesquisa, que teve como objetivo identificar os fatores associados ao desenvolvimento mental e motor de lactentes que frequentam creches da rede municipal do Recife.

\section{Métodos}

Este estudo transversal foi conduzido em quatro das sete creches municipais do Distrito Sanitário IV da cidade do Recife, que atendem crianças na faixa etária de interesse. As crianças que frequentam essas creches apresentam condições socioeconômicas semelhantes e recebem o mesmo padrão de assistência da Secretaria de Educação do município em relação ao currículo pedagógico, dieta e assistência à saúde.

A amostra foi composta por todas as crianças que tinham entre quatro e 24 meses de idade no momento da avaliação e que frequentavam regularmente as referidas creches. Foram excluídas do estudo três crianças que não cooperaram durante a execução da escala de desenvolvimento infantil de Bayley, impedindo a sua realização, bem como três crianças com déficits neurológicos acentuados (paralisia cerebral, deficiência mental e déficit sensorial grave) detectados no momento da avaliação; a amostra final, portanto, foi constituída por 108 crianças. A coleta de dados foi realizada no período de fevereiro a abril de 2005.

Os testes de avaliação do desenvolvimento neuropsicomotor foram efetuados nas creches, em uma sala de exame apropriada, por duas pesquisadoras com formação em desenvolvimento infantil. Foram utilizadas as subescalas mental e motora da Escala de Desenvolvimento Infantil de Bayley versão II (BSID-II) ${ }^{(19)}$, que tem por objetivo detectar atrasos no desenvolvimento de crianças de um a 42 meses, servindo de base para o planejamento de intervenções precoces. Os escores obtidos em cada subescala são convertidos em índices que apresentam uma média de 100 e um desvio padrão de 15 pontos, sendo que valores entre 85 e 115 são considerados dentro da variação de normalidade. $\mathrm{O}$ controle de qualidade da execução dos testes foi feito por meio da observação em um mesmo teste concomitantemente por duas avaliadoras, em $16 \%$ dos testes. O coeficiente de correlação de Pearson, utilizado na avaliação da concordância dos coeficientes entre observadores, foi alto para ambas as escalas $(r \geq 0,90)$.

As avaliações socioeconômica, demográfica e biológica das crianças foram realizadas nas creches, por meio de entrevistas com as mães, aplicando-se um questionário específico com perguntas fechadas acerca das características socioeconômicas das famílias (renda, nível educacional e idade materna, condições de moradia, coabitação) e informações relacionadas às crianças (sexo, idade gestacional, peso ao nascer, idade $\mathrm{e}$ ocorrência de hospitalização).

O peso e o comprimento das crianças foram medidos por duas antropometristas treinadas, utilizando-se técnicas e equipamentos padronizados de acordo com procedimentos estabelecidos pela Organização Mundial de Saúde (OMS) ${ }^{(20)}$. $\mathrm{O}$ estado nutricional foi avaliado pelos índices peso/idade, comprimento/idade e peso/comprimento, expressos em média de escore Z, de acordo com o padrão de referência da OMS ${ }^{(21)}$. Os índices antropométricos foram categorizados em $<-2$ escores $Z$ 
como desnutrição, $\geq-2$ e $<-1$ escore $Z$ como risco nutricional e $\geq-1$ escore $Z$ como eutrofia.

A concentração de hemoglobina foi avaliada por duas técnicas treinadas mediante análise de uma amostra de sangue capilar, utilizando-se um hemoglobinômetro portátil (HemoCue Ltd., Sheffield, UK). Adotou-se o nível de hemoglobina abaixo de $11 \mathrm{~g} / \mathrm{dL}$ como definição de anemia ${ }^{(22)}$.

Todos os questionários foram pré-codificados e avaliados regularmente quanto à consistência do preenchimento. Realizou-se entrada dupla de dados no programa estatístico Epi-Info, versão 6,04 (CDC, Atlanta) para checar erros de digitação. Este programa também foi utilizado para a análise estatística. A classificação do estado nutricional foi realizada com o programa WHO Anthro, versão 2,0.

As variáveis de desfecho (índices de desenvolvimento mental e motor) foram analisadas como contínuas. Empregou-se o teste $t$ e a Análise de Variância (ANOVA), quando apropriado, para comparar as médias, adotando-se $p \leq 0,05$ como significante.

Este estudo foi aprovado pelo Comitê de Ética em Pesquisa do Hospital Oswaldo Cruz. As mães das crianças participantes foram informadas sobre os objetivos e procedimentos da pesquisa e assinaram o termo de consentimento livre e esclarecido antes da inclusão no estudo. Foram encaminhados para os serviços de saúde da rede municipal do Recife ou centros de reabilitação especializados os lactentes com déficit nutricional, anemia grave ou déficit neurológico.

\section{Resultados}

A média do índice de desenvolvimento mental (IDM) das crianças estudadas foi $88,2 \pm 11,3$ pontos e a do índice de desenvolvimento motor (IDP) foi 94,7 $\pm 12,4$ pontos (Tabela 1 ). Verificou-se uma distribuição homogênea das crianças em relação à idade, com cerca da metade delas na faixa etária entre quatro e 12 meses de idade.

As características da amostra estão apresentadas nas Tabelas 2 e 3 . Verificou-se que as crianças pertenciam a famílias com baixa condição socioeconômica, uma vez que $70,5 \%$ delas tinham renda mensal per capita $\leq 1 / 4$ de salário mínimo. Embora a população fosse muito pobre, $66,7 \%$ das mães estudaram pelo menos cinco a 11 anos. Aproximadamente metade delas estava na faixa etária inferior a 26 anos e apenas sete $(6,5 \%)$ eram adolescentes. Chama atenção as condições de habitação: 79,6\% das famílias tinham água encanada em casa, porém só a metade possuía sanitário com descarga (Tabela 2).

A Tabela 3 mostra que, entre as crianças estudadas, $48,1 \%$ eram do sexo masculino, com uma proporção de nascidos com baixo peso e de parto pré-termo de 9,3\%. Quanto ao estado nutricional, a proporção de crianças em risco nutricional e desnutridas (<-1 escore $Z$ ) foi de $12,1 \%$ para o índice peso/ idade, 35,4\% para o índice comprimento/idade e 10,1\% para o índice peso/comprimento. Desnutrição (índices antropométricos $<-2$ escores $Z$ ) foi observada em apenas $1 \%$ das crianças avaliadas pelo índice peso/idade e 3,0\% pelo índice peso/comprimento, frequência que aumentou para $12,1 \%$ pelo índice comprimento/idade. Aproximadamente 66\% delas tinham nível de hemoglobina $<11 \mathrm{~g} / \mathrm{dL}$, no entanto, apenas $7 \%$ apresentaram anemia moderada a grave (nível de hemoglobina abaixo de $9 \mathrm{~g} / \mathrm{dL}$ ).

Não se observou significância estatística entre as condições socioeconômicas e demográficas e o desenvolvimento mental e motor (Tabela 2). Verifica-se, na Tabela 3, que as crianças nascidas com baixo peso apresentaram média do índice de desenvolvimento mental e motor aquém daquelas nascidas com peso $\geq 2500 \mathrm{~g}$, com diferença estatística apenas para o desenvolvimento motor $(p=0,05)$. A mesma tendência foi observada para as crianças que nasceram prematuras, com uma diferença significativa tanto para o índice de desenvolvimento mental $(p=0,05)$ como o do desenvolvimento motor $(p=0,004)$. Com relação ao estado nutricional atual, as crianças em risco nutricional e desnutridas, quando avaliadas pelo índice peso/comprimento, apresentaram a média do índice de desenvolvimento motor significativamente mais baixa em comparação às crianças eutróficas $(p=0,04)$.

\section{Discussão}

Em populações menos favorecidas economicamente, a creche costuma ser uma importante opção de cuidado alternativo para as crianças em idade pré-escolar. Contudo, o

Tabela 1 - Índices de desenvolvimento mental e motor de crianças em creches municipais do Recife, 2005

\begin{tabular}{lcccc}
\hline & $\mathbf{n}$ & Média & dp & IC95\% \\
\hline Desenvolvimento mental & 108 & 88,2 & 11,3 & $86,1-90,3$ \\
Desenvolvimento motor & 108 & 94,7 & 12,4 & $92,4-97,0$ \\
\hline
\end{tabular}

dp: desvio padrão; IC: intervalo de confiança. 
Tabela 2 - Índices de desenvolvimento mental (IDM) e motor (IDP) segundo as características socioeconômicas e demográficas das crianças em creches municipais do Recife, 2005

\begin{tabular}{|c|c|c|c|c|c|c|}
\hline & $\mathrm{n}$ & $\%$ & IDM & Valor de $p$ & IDP & Valor de $p$ \\
\hline \multicolumn{7}{|l|}{ Renda familiar per capita (SM)* } \\
\hline$\leq 0,25$ & 67 & 70,5 & 87,8 & 0,62 & 93,4 & 0,27 \\
\hline$>0,25$ & 28 & 29,5 & 89,0 & & 96,5 & \\
\hline \multicolumn{7}{|l|}{ Escolaridade materna (anos) } \\
\hline $1-4$ & 36 & 33,3 & 88,2 & 0,98 & 94,3 & 0,80 \\
\hline $5-11$ & 72 & 66,7 & 88,2 & & 94,9 & \\
\hline \multicolumn{7}{|l|}{ Idade materna (anos) } \\
\hline $17-25$ & 52 & 48,1 & 88,5 & 0,81 & 95,4 & 0,58 \\
\hline$\geq 26$ & 56 & 51,9 & 88,0 & & 94,0 & \\
\hline \multicolumn{7}{|l|}{ Coabitação } \\
\hline Sim & 55 & 50,9 & 89,1 & 0,44 & 94,0 & 0,56 \\
\hline Não & 53 & 49,1 & 87,4 & & 95,4 & \\
\hline \multicolumn{7}{|l|}{ Tamanho da família } \\
\hline$\leq 4$ & 54 & 50,0 & 88,5 & 0,8 & 94,6 & 0,94 \\
\hline$\geq 5$ & 54 & 50,0 & 87,9 & & 94,8 & \\
\hline \multicolumn{7}{|l|}{ Crianças $<5$ anos } \\
\hline 1 & 41 & 38,0 & 88,3 & 0,97 & 95,7 & 0,51 \\
\hline$\geq 2$ & 67 & 62,0 & 88,2 & & 94,1 & \\
\hline \multicolumn{7}{|l|}{ Tipo de moradia } \\
\hline Casa/apartamento & 86 & 79,6 & 88,2 & 0,97 & 94,8 & 0,82 \\
\hline Quarto/cômodo/outros & 22 & 20,4 & 88,1 & & 94,1 & \\
\hline \multicolumn{7}{|l|}{ Tipo de teto } \\
\hline Laje de concreto & 24 & 22,3 & 87,3 & 0,82 & 92,4 & 0,32 \\
\hline Telha de barro & 40 & 37,0 & 88,0 & & 93,7 & \\
\hline Telha de cimento/amianto & 44 & 40,7 & 89,0 & & 96,8 & \\
\hline \multicolumn{7}{|l|}{ Água encanada } \\
\hline Sim & 86 & 79,6 & 87,7 & 0,34 & 95,2 & 0,40 \\
\hline Não & 22 & 20,4 & 90,3 & & 92,7 & \\
\hline \multicolumn{7}{|l|}{ Sanitário } \\
\hline Com descarga & 56 & 51,8 & 87,2 & 0,63 & 94,5 & 0,74 \\
\hline Sem descarga & 38 & 35,2 & 89,2 & & 94,1 & \\
\hline Sem sanitário & 14 & 13,0 & 89,6 & & 97,0 & \\
\hline \multicolumn{7}{|l|}{ Geladeira } \\
\hline Sim & 71 & 65,7 & 88,3 & 0,88 & 94,8 & 0,91 \\
\hline Não & 37 & 34,3 & 88,0 & & 94,5 & \\
\hline \multicolumn{7}{|l|}{ Rádio } \\
\hline Sim & 57 & 52,8 & 88,1 & 0,89 & 96,2 & 0,18 \\
\hline Não & 51 & 47,2 & 88,4 & & 93,0 & \\
\hline \multicolumn{7}{|l|}{ Televisão } \\
\hline Sim & 92 & 85,2 & 88,0 & 0,58 & 95,1 & 0,41 \\
\hline Não & 16 & 14,8 & 89,7 & & 92,3 & \\
\hline
\end{tabular}

*13 casos sem informação; SM: salário mínimo. 
Tabela 3 - Índices de desenvolvimento mental (IDM) e motor (IDP) segundo as características biológicas de crianças em creches municipais do Recife, 2005

\begin{tabular}{|c|c|c|c|c|c|c|}
\hline & $\mathrm{n}$ & $\%$ & IDM & Valor de $p$ & IDP & Valor de $p$ \\
\hline \multicolumn{7}{|l|}{ Sexo } \\
\hline Masculino & 52 & 48,1 & 87,7 & 0,62 & 95,3 & 0,61 \\
\hline Feminino & 56 & 51,9 & 88,8 & & 94,1 & \\
\hline \multicolumn{7}{|c|}{ Peso ao nascer $(\mathrm{g})^{\star}$} \\
\hline$\leq 2499$ & 9 & 9,3 & 83,9 & 0,35 & 84,7 & 0,05 \\
\hline 2500-2999 & 16 & 16,5 & 90,9 & & 96,1 & \\
\hline$\geq 3000$ & 72 & 74,2 & 88,0 & & 95,4 & \\
\hline \multicolumn{7}{|c|}{ Idade gestacional (meses) } \\
\hline 7 a 8 & 10 & 9,3 & 81,5 & 0,05 & 84,0 & 0,004 \\
\hline 9 & 98 & 90,7 & 88,9 & & 95,8 & \\
\hline \multicolumn{7}{|c|}{ Peso/idade (escore Z) ${ }^{\star *}$} \\
\hline$<-1$ & 12 & 12,1 & 89,4 & 0,64 & 90,5 & 0,23 \\
\hline$\geq-1$ & 87 & 87,9 & 87,8 & & 95,0 & \\
\hline \multicolumn{7}{|c|}{ Comprimento/idade (escore Z) ${ }^{\star *}$} \\
\hline$<-1$ & 35 & 35,4 & 87,6 & 0,76 & 93,1 & 0,40 \\
\hline$\geq-1$ & 64 & 64,6 & 88,3 & & 95,2 & \\
\hline \multicolumn{7}{|c|}{ Peso/comprimento (escore Z) ** } \\
\hline$<-1$ & 10 & 10,1 & 88,3 & 0,93 & 87,2 & 0,04 \\
\hline$\geq-1$ & 89 & 89,9 & 88,0 & & 95,3 & \\
\hline \multicolumn{7}{|c|}{ Nível de hemoglobina $(\mathrm{g} / \mathrm{dL})^{* *}$} \\
\hline$<11,0$ & 65 & 65,7 & 88,6 & 0,47 & 93,4 & 0,23 \\
\hline$\geq 11,0$ & 34 & 34,3 & 86,9 & & 96,5 & \\
\hline \multicolumn{7}{|c|}{ Hospitalização anterior } \\
\hline Sim & 43 & 39,8 & 86,5 & 0,21 & 94,0 & 0,67 \\
\hline Não & 65 & 60,2 & 89,3 & & 95,1 & \\
\hline
\end{tabular}

*11 casos sem informação; **9 casos sem informação.

seu papel em relação ao desenvolvimento infantil permanece controverso $^{(11-18,23)}$.

Apesar de a população aqui estudada ser pobre e viver em condições ambientais adversas, com a maioria das famílias com renda mensal per capita inferior a meio salário mínimo, as crianças apresentaram os índices de desenvolvimento dentro do limite da normalidade. No entanto, os índices de desenvolvimento mental e motor ficaram 11 e 6 pontos, respectivamente, abaixo da média da população de referência, diferenças correspondentes a cerca de meio desvio padrão, valor considerado de impacto em saúde pública ${ }^{(24)}$.

Este é um estudo descritivo realizado com uma amostra constituída, por conveniência, por um grupo de crianças de até 24 meses, residentes em uma área pobre da cidade do Recife e que frequentavam quatro creches municipais. A falta de um grupo controle composto por crianças pertencentes à mesma comunidade, porém não frequentadoras de creches, não permite estabelecer associação entre a frequência à creche e o desenvolvimento infantil. Entretanto, observou-se, nas creches estudadas, uma grande concentração de crianças nas salas, contrastando com um número restrito de educadoras. Essa sobrecarga de trabalho faz com que as educadoras passem a se ocupar mais com os cuidados básicos de higiene e alimentação. Esse fato, somado a uma carência de conhecimentos sobre técnicas de estimulação do desenvolvimento infantil, pode comprometer a qualidade da estimulação. 
Um dos aspectos positivos a ser considerado no presente estudo é o instrumento utilizado na avaliação do desenvolvimento. A Escala de Bayley é considerada padrão-ouro para avaliar o desenvolvimento infantil, mas são escassas as pesquisas nacionais e internacionais que utilizaram esta escala em crianças de creches. Foram identificados apenas dois estudos brasileiros, ambos no estado de São Paulo, que utilizaram a Escala de Bayley para avaliar o desenvolvimento de crianças em creches públicas e, em ambos, observou-se também uma tendência a atraso no desenvolvimento mental e motor em crianças atendidas em creches ${ }^{(11,16)}$.

No interior do estado de Pernambuco, foi realizada uma série de estudos sobre o desenvolvimento infantil com crianças de baixa renda por meio da Escala de Bayley $\mathrm{I}^{(5,24-}$ 26), notando-se escores de desenvolvimento mental e motor mais elevados do que os evidenciados na nossa amostra, mesmo que a última se refira a uma população urbana de nível socioeconômico e idade semelhantes. Apesar de o desenho do presente estudo não permitir avaliar a associação da frequência à creche com o desenvolvimento infantil, uma possível hipótese para explicar essa diferença seria o fato de que as crianças do interior de Pernambuco, como não frequentavam creches, permaneciam em seus domicílios, fortalecendo o vínculo entre mãe e criança. Além disso, no domicílio, a proporção adulto/criança é em geral menor do que na creche, propiciando contato mais próximo da criança com uma pessoa de referência, o que é importante para o desenvolvimento infantil.

Em relação à pobreza e sua repercussão sobre o desenvolvimento infantil, a maioria dos autores tem apontado em suas pesquisas uma importante associaçãa ${ }^{(6,7,25,27,28)}$. Em nosso estudo, não se observou associação significativa entre as variáveis socioeconômicas e o desenvolvimento infantil, provavelmente em virtude do nível generalizado e homogêneo de pobreza da população estudada, não havendo o contraste necessário para demonstrar tal diferença.

Dentre as condições socioeconômicas, o baixo grau de escolaridade materna se destaca na literatura como importante fator de risco para o atraso no desenvolvimento infantil, já que está intimamente relacionado à qualidade da estimulação ambiental ${ }^{(1,2,4,29)}$. No presente estudo, a escolaridade materna não se associou ao desenvolvimento infantil talvez pelo fato de as crianças permanecerem a maior parte do tempo na creche, diminuindo a influência dos fatores relacionados ao ambiente domiciliar.

Com relação às características biológicas, na presente amostra foi observada uma associação significativa entre desenvolvimento mental e idade gestacional e entre desenvolvimento motor e idade gestacional, peso ao nascer e estado nutricional atual, avaliado pelo índice peso/comprimento.

Apesar do tamanho reduzido da amostra, com apenas dez crianças prematuras, pôde-se verificar uma associação entre a prematuridade e o atraso do desenvolvimento tanto mental como motor, dado concordante com a literatura. O desenvolvimento dos prematuros deve ser cuidadosamente monitorizado para garantir que essas crianças recebam adequado suporte e intervenção, a fim de melhorar seu prognóstico ${ }^{(30,31)}$.

As crianças nascidas com baixo peso também apresentaram índice médio de desenvolvimento motor significativamente mais baixo quando comparadas às crianças nascidas com peso insuficiente ou adequado. Os nascidos a termo com baixo peso constituem um grupo de risco para o atraso do desenvolvimento durante a infância, bem como para uma elevada morbimortalidade infantil. Por isso, a restrição de crescimento intrauterina deve ser considerada um relevante problema de saúde pública, especialmente em países em desenvolvimento, onde a população é consideravelmente mais pobre e suscetível a múltiplos fatores de risco biológicos e ambientais ${ }^{(5,25,26,32)}$.

Não se evidenciou prevalência elevada de crianças com desnutrição, mesmo reconhecendo que o índice comprimento/idade $<-2$ escores $Z$ esteve presente em percentual elevado $(12 \%)$. No entanto, verificou-se uma tendência significativa para redução da média do índice de desenvolvimento motor em crianças em risco nutricional e desnutridas avaliadas pelo índice peso/comprimento, que é um marcador de desnutrição aguda. Esse achado também foi encontrado em estudos realizados com crianças na mesma faixa etária de países em desenvolvimento ${ }^{(25,33)}$. Os mecanismos pelos quais a desnutrição se associa ao atraso no desenvolvimento não são claros, mas alguns fatores têm sido postulados, como alterações estruturais e bioquímicas no cérebro, redução na atividade motora infantil e/ou alteração do seu humor ${ }^{(34)}$.

Neste estudo, apesar de as crianças apresentarem elevado percentual de anemia, essa enfermidade não se associou aos índices de desenvolvimento mental ou motor. Há evidências atuais na literatura de que a anemia ferropriva funciona como um indicador de risco para atraso no desenvolvimento e deve ser controlada, especialmente em populações com múltiplos fatores de risco ${ }^{(25,34)}$.

Em virtude do exposto, pode-se concluir que, nas creches estudadas, as crianças apresentaram desenvolvimento neuropsicomor ainda dentro da faixa de normalidade, de 
acordo com o instrumento utilizado, mas aquém do esperado para a faixa etária, em comparação a populações de países desenvolvidos. Os fatores associados ao baixo desempenho do desenvolvimento nessa população estão relacionados a variáveis biológicas, destacando-se os déficits nutricionais, cuja redução deve ser uma prioridade para gestores em saúde pública e educação.

\section{Referências bibliográficas}

1. Walker SP, Wachs TD, Gardner JM, Lozoff B, Wasserman GA, Pollitt E et al. Child development: risk factors for adverse outcomes in developing countries. Lancet 2007;369:145-57.

2. Weisglas-Kuperus N, Baerts W, Smrkovsky M, Sauer PJ. Effects of biological and social factors on the cognitive development of very low birth weight children. Pediatrics 1993;92:658-65.

3. de Andraca I, Pino P, de la Parra A, Rivera F, Castillo M. Risk factors for psychomotor development among infants born under optimal biological conditions. Rev Saude Publica 1998;32:138-47.

4. Halpern R, Giugliani ER, Victora CG, Barros FC, Horta BL. Risk factors for suspicion of developmental delays at 12 months of age. J Pediatr (Rio J) 2000;76:421-8.

5. Eickmann SH, Lira PI, Lima MC. Mental and motor development at 24 months of full-term low birthweight infants. Arq Neuropsiquiatr 2002;60:748-54.

6. Chilton M, Chyatte M, Breaux J. The negative effects of poverty \& food insecurity on child development. Indian J Med Res 2007;126:262-72.

7. Guo G, Harris KM. The mechanisms mediating the effects of poverty on children's intellectual development. Demography 2000;37:431-47.

8. Segall-Corrêa AM, Gonçalves NN, Chalita LV, Russo-Leite GP, Padovani CR, Gonçalves A. Factors determining weight and height in children aged 3 months to 6 years enrolled in a public municipal day-care center in Brazil. Rev Panam Salud Publica 2002;12:19-25.

9. Bueno MB, Marchioni DM, Fisberg RM. Changes in the nutritional status of children in public day care facilities in the municipality of São Paulo, Brazil. Rev Panam Salud Publica 2003;14:165-70.

10. Rocha DS, Lamounier JA, Capanema FD, Franceschini SC, Norton RC, Costa $A B$ et al. Nutritional status and anemia prevalence in children enrolled at day care centers in Belo Horizonte, Minas Gerais, Brazil. Rev Paul Pediatr 2008;26:6-13.

11. Paula CS. Atrasos de desenvolvimento mental e motor em crianças de creches de comunidade urbana de baixa renda e fatores de risco associados [dissertação de mestrado]. São Paulo (SP): Unifesp; 2001.

12. Wasik BH, Ramey CT, Bryant DM, Sparling JJ. A longitudinal study of two early intervention strategies: Project CARE. Child Dev 1990;61:1682-96.

13. Caughy MO, DiPietro JA, Strobino DM. Day-care participation as a protective factor in the cognitive development of low-income children. Child Dev 1994:65:457-71.

14. Burchinal MR, Roberts JE, Nabors LA, Bryant DM. Quality of center child care and infant cognitive and language development. Child Dev 1996;67:606-20.

15. Broberg AG, Wessels H, Lamb ME, Hwang CP. Effects of day care on the development of cognitive abilities in 8-year-olds: a longitudinal study. Dev Psychol 1997;33:62-9.

16. de Miranda CT, de Paula CS, Palma D, da Silva EM, Martin D, de Nóbrega FJ. Impact of the application of neurolinguistic programming to mothers of children enrolled in a day care center of a shantytown. São Paulo Med J 1999;117:63-71.

17. RapoportA, Piccinini CA. O ingresso e adaptação de bebês e crianças pequenas à creche: alguns aspectos críticos. Psicol Reflex Crit 2001;14:81-95.

\section{Agradecimentos}

Às famílias participantes, aos assistentes de pesquisa, aos funcionários das creches e ao Conselho Nacional de Desenvolvimento Científico e Tecnológico (CNPq) pelo financiamento a esta pesquisa e pelas Bolsas de Produtividade em Pesquisa dos Professores Marília Lima e Pedro Lira.

18. Brooks-Gunn J, Han WJ, Waldfogel J. Maternal employment and child cognitive outcomes in the first three years of life: the NICHD Study of Early Child Care. Child Dev 2002;73:1052-72.

19. Bayley N. The Bayley Scales of Infant Development. 2nd ed. (BSID II). San Antonio (TX): The Psychological Corporation; 1993.

20. WHO Expert Committee on Physical Status. The use and interpretation of anthropometry: report of a WHO Expert Committee - World Health Organization technical report series 854. Geneva: World Health Organization; 1995.

21. WHO Multicentre Growth Reference Study Group. WHO Child Growth Standards: length/height-for-age, weight-for-age, weight-for-length, weightfor-height and body mass index-for-age: methods and development. Geneva: World Health Organization; 2006.

22. DeMaeyer EM, Dallman P, Gurney JM, Hallberg L, Sood SK, Srikantia SG. Preventing and controlling iron deficiency anaemia through primary health care: a guide for health administrators and programme managers. Geneva: World Health Organization; 1989.

23. Guralnick MJ. Effectiveness of early intervention for vulnerable children: a developmental perspective. Am J Mental Retard 1998;102:319-45.

24. Eickmann SH, Lima AC, Guerra MQ, Lima MC, Lira PI, Huttly SR et al. Improved cognitive and motor development in a community-based intervention of psychosocial stimulation in northeast Brazil. Dev Med Child Neurol 2003;45:536-41.

25. Lima MC, Eickmann SH, Lima AC, Guerra MQ, Lira PI, Huttly SR et al. Determinants of mental and motor development at 12 months in a low income population: a cohort study in northeast Brazil. Acta Paediatr 2004;93: 969-75.

26. Morris SS, Grantham-McGregor SM, Lira PI, Assunção AM, Ashworth A. Effect of breastfeeding and morbidity on the development of low birthweight term babies in Brazil. Acta Paediatr 1999;88:1101-6.

27. Huston AC, McLoyd VC, Coll GC. Children and poverty: issues in contemporary research. Child Dev 1994;65:275-82.

28. Dearing E. Psychological costs of growing up poor. Ann NY Acad Sci 2008;1136:324-32.

29. Garrett P, Ng'andu N, Ferron J. Poverty experiences of young children and the quality of their home environments. Child Dev 1994;65:331-45.

30. Rugolo LM. Growth and developmental outcomes of the extremely preterm infant. J Pediatr (Rio J) 2005;81 (Suppl 1):101-10.

31. Allen MC. Neurodevelopmental outcomes of preterm infants. Curr Open Neurol 2008;21:123-8.

32. Grantham-McGregor SM, Lira PI, Ashworth A, Morris SS, Assunção AM. The development of low birth weight term infants and the effects of the environment in Northeast Brazil. J Pediatr 1998;132:661-6.

33. Gardner JM, Grantham-McGregor SM, Himes J, Chang S. Behaviour and development of stunted and nonstunted Jamaican children. J Child Psychol Psychiatry 1999;40:819-27.

34. Lozoff B. Considering environmental factors in research on nutrient deficiencies and infant development. Clinical trials in infant nutrition. Nestlé Nutr Worksh Series 1998;40:203-18. 